\title{
Water quality and enrichment of sedimentary polycyclic aromatic hydrocarbons (PAHs) relation to fish culture in Malaysia
}

\begin{abstract}
This study investigated the potential effects of the fish aquaculture on water quality and enrichment of PAHs in the aquaculture surface sediments. Water quality parameters and PAHs were determined at fish farms and reference sites. A significant decrease in dissolved oxygen at fish farms was observed $(\mathrm{p}<0.05)$. Enrichment of total organic carbons (TOC) and PAHs in comparison to reference site were significant $(\mathrm{p}<0.05)$. Enrichment of TOC had significant correlation with enrichment of PAHs $(\mathrm{p}<0.05)$ especially carcinogenic PAHs. Cluster and principle components analyses revealed an additional source of PAHs (35\%) into the fish farm sediments. The finding of this study indicated fish farming is a significant source of dissolved oxygen deterioration and enrichment of PAHs. Therefore an appropriate monitoring system is required for the sustainable development of fish farming sector.
\end{abstract}

Keyword: Fish farms; Dissolved oxygen; Enrichments; Polycyclic aromatic hydrocarbons; Total organic carbons 